\title{
BINARY INTERACTIONS IN STAR CLUSTERS
}

\author{
Stephen L. W. McMillan \\ Northwestern University
}

Over the past decade, a very considerable amount of effort in stellar dynamics has gone into the study of interactions between binary systems and other stars. The asymptotic analytic results obtained by Heggie (1975) for binary-single star encounters have been largely confirmed and extended by later numerical experiments (Hills 1975, Hut and Bahcall 1983). Binary-binary interactions have been studied by Mikkola (1983).

In numerical simulations of real star clusters, it is found that binaries can not always be regarded as interacting with only one other star or binary at a time. When the number of stars in the core of a stellar system is small, statistical fluctuations about mean-field quantities can be very large, so that all higher-order interactions tend to "turn on" almost simultaneously with the first appearance of triple effects. These interactions are very difficult to treat theoretically and are currently quite poorly understood. While $\mathrm{N}$-body experiments offer an obvious way of studying binaries "in the field," their use still presents some difficulties. In an isolated, self-gravitating system containing a small number of bodies, the first hard binary to form usually comes to dominate the dynamics of the cluster and cause it to expand or even dissolve, severely limiting the number of binary interactions that can be studied. Accounts of the behavior of binaries in such circumstances tend to be largely anecdotal in nature.

I present here a quantitative account of over one thousand "close" encounters between binaries and other stars during the final stages of globular cluster core collapse. The hybrid integration is described in detail by McMillan (1986, "run 2"). Because the $\mathrm{N}$-body system is only a small part of an extensive confining cluster, the large amount of energy liberated by hard binaries is relatively easily absorbed, allowing densities to remain high and conditions to remain suitable for the study of binary encounters over long periods of time. The run described here lasted for some 800 initial core relaxation times, corresponding to around 5500 internal time units. Every half time unit, the identity, binding energy (B), eccentricity, center of mass energy (E) and nearest neighbors of all significant bound pairs (i.e. those having binding energy greater than or comparable to the mean stellar kinetic energy, $1.5 \mathrm{kT}$ ) were stored for subsequent analysis. In addition, similar information on close triple systems involving the center of mass of any close pair (bound or unbound) was retained.

The sampling time interval is longer than most binary interactions, allowing them to be convenienty identified simply by checking for abrupt changes in a binary's orbital parameters or components. Once a significant encounter is found, the star(s) involved are easily determined from the stored data, and the event is classified according to the binding energy change $(\delta \mathrm{B})$, the total number of stars involved (I), the local core conditions, and so on. A star is deemed an "interactor" if it is a component of, or a neighbor within five semi-major axes of, the binary before 
or after the interaction. For the small number of cases in which no obvious interactor is found, a value of $I=3$ is assumed.

The overall statistics of the run, for encounters resulting in $|\delta \mathrm{B}|>\mathrm{kT}$, are illustrated in the table below, which lists the number of interactors found in various situations. In the first four columns, they are broken down by type of interaction (binary creation or destruction, flyby or exchange). In the next two, the binary's environment is taken into account, the local density $\rho$ being divided into two ranges: "low" (less than five times the initial density) and "high" (near the peak density at core collapse, some ten to twenty times higher). During the course of the run, the system oscillated between the high- and low-density states approximately eight times as binary formation, heating and ejection governed the core dynamics. The final two columns subdivide the encounters by the average binding energy of the binary involved.

$\begin{array}{lcccccccc} & \text { Creation } & \text { Destruction } & \text { Flyby } & \text { Exchange } & \text { Low- } \rho & \text { High- } \rho & \text { B } \leq 8 \text { kT } & \text { B > 8 kT } \\ \mathrm{I}=3 & 135 & 207 & 271 & 195 & 535 & 273 & 554 & 254 \\ \mathrm{I}=4 & 60 & 104 & 81 & 84 & 166 & 163 & 295 & 34 \\ \mathrm{I} \geq 5 & 36 & 64 & 58 & 56 & 105 & 109 & 194 & 20\end{array}$

Evidently a substantial fraction $(\sim 40 \%)$ of all binary encounters involve more than just three stars. For low-energy binaries or high ambient densities, the fraction is even higher $(\sim 50 \%)$. As expected, hard binaries tend to interact with just one star at a time, since a perturber has to approach within a few binary semi-major axes before a strong triple interaction can take place.

The value of $I$ also affects the detailed statistical properties of the interactions. For relatively soft binaries $(B<20 \mathrm{kT})$, the distribution of $\Delta \equiv \delta B / B$ for $I=3$ is significantly different from that for I $\geq 4$, while the expected correlation between $\delta \mathrm{B}$ and $\delta \mathrm{E}$, the binary's recoil energy, is almost entirely absent in the latter case, as the number of ways in which the recoil energy can be distributed increases. Even for triple encounters involving hard binaries, the $\Delta$ distribution is not consistent with the $(1+\Delta)^{-9 / 2}$ behavior found by Heggie, and $\delta E$ falls well below the predicted value of $\delta \mathrm{B} / 3$ for $\delta \mathrm{B}>40 \mathrm{kT}$. This may indicate that a considerable fraction of the "triples" are actually misclassified higher-order encounters. (Alternatively, these encounters might not have been completely over when sampled: proximity to a third star would tend to lower the calculated center of mass energy.)

The results of this preliminary investigation suggest that a sizeable fraction of all binary interactions in a star cluster actually involve four or more participants. As the binding energy of a binary increases, it becomes more likely that it will interact with just one other star at a time, but even hard binary encounters appear to be appreciably affected by the presence of additional nearby perturbers. As a result, such encounters may be somewhat more efficient at directly heating the cluster than was previously thought. At lower energies, extra participants in an interaction can substantially alter the distribution of the fractional binding energy change, and reduce almost to zero the correlation between binding energy change and binary recoil energy.

Note that these results refer to the specific problem of globular cluster core collapse, where velocities are somewhat higher (relative to the central potential) than for an isolated, self-bound system. It is not immediately clear how to scale the above conclusions to the latter situation. The routine accumulation of similar data in such integrations would be a welcome development.

\section{REFERENCES}

Heggie, D. C. 1975 Monthly Notices Roy. Astron. Soc. 173, 729. Hills, J. G. 1975 Astrophys. J. 80, 809. Hut, P. and Bahcall, J. N. 1983 Astrophys. J. 268, 319. McMillan, S. L. W. 1986 Astrophys. J. 307, 261. Mikkola, S. 1983 Monthly Notices Roy. Astron. Soc. 205, 733. 